\title{
Process and Structure of Electromagnetic Shielding Plywood Composite Laminated with Carbon Fiber Paper
}

\author{
Quanping Yuan, Keyang Lu and Feng Fu ${ }^{*}$
}

\author{
Research Institute of Wood Industry, Chinese Academy of Forestry; Key Lab of Wood Science and Technology of State \\ Forestry Administration, Beijing, 100091, P.R. China
}

\begin{abstract}
Carbon fiber paper (CFP), a type of flexible planer electromagnetic shielding material with thin thickness, low density, and good adhesion property and permeability, was laminated with wood veneer to produce a plywood composite with good shielding effectiveness (SE). It was found that a hot-press pressure of 1.2 MPa and a double-sized adhesive concentration of $380 \mathrm{~g} / \mathrm{m}^{2}$ were most appropriate for the production of CFP plywood composites. SE of plywood composite laminated with single layer CFP was better than that before hot pressed, which result from the formation of three-dimensional and smaller conductive carbon fiber circuitries. The space between two-layer CFPs and the thickness of the surface-layer veneer had significant influence on SE. The SE of the composites laminated with two-layer CFPs was significantly higher than those with one-layer CFPs. The SE in the frequency range of $30 \mathrm{MHz}$ to $1 \mathrm{GHz}$ reached above $30 \mathrm{~dB}$ depending on the space between two-layer CFPs and the thickness of the surface veneer, which was sufficient SE for commercialization and the use of plywood composites.
\end{abstract}

Keywords: Carbon fiber paper, electromagnetic shielding, hot-press process, laminate structure, plywood composite.

\section{INTRODUCTION}

Wood is one of the most abundant biological materials, and timber products have been widely used in both structural and nonstructural applications such as furniture and building construction. However, timber products remain low-value added materials, especially for the products of fast-growing plantation wood, and many physical technologies have been attempted for the production of higher-value added products for the industry. One of the methods is to modify wood products in terms of their electromagnetic shielding functionality, and thus replace expensive and heavy metal shielding materials. This wooden functional material could also be used as shielding material to reduce harmful electromagnetic interference in the fields of military, precision instruments, and hospital equipment.

Electromagnetic shielding wooden composites have been studied for about 30 years, with concentration mainly on combining the conductive shielding with wooden elements and/or by high-temperature carbonization of wood constituents. Wood particles were coated with a layer of nickel by electroless plating technology [1-3], and the coated particles were then used for the production of particleboards, the SE of which could reach $40 \mathrm{~dB}$ in the frequency range of $10 \mathrm{MHz}$ to $1 \mathrm{GHz}$ [4]. Veneer has also been electrolessplated with nickel on its surface, and can reach an SE of 55$60 \mathrm{~dB}$ in the frequency range of $9 \mathrm{kHz}$ to $1.5 \mathrm{GHz}[5,6]$. The plywood made with the modified veneers by the

*Address correspondence to this author at the Research Institute of Wood Industry, Chinese Academy of Forestry; Key Lab of Wood Science and Technology of State Forestry Administration, Xiangshan Road, Haidian District, Beijing, 100091, P.R. China; Tel: 86-13683598407, 86-1062889973; Fax: 86-10-62881937; E-mails: cafst@163.com, feng@caf.ac.cn $\gamma$-aminopropyltri-hydroxysilane (APTHS) before being plated with nickel could achieve an SE of higher than $60 \mathrm{~dB}$ in the frequency range of $10 \mathrm{MHz}$ to $1.5 \mathrm{GHz}$ [7].

Metal nets [8], metal fiber [9-11], and conductive powder [12] have also been mixed with wooden materials to prepare shielding composites. Among them, the fiberboard versions with two layers of 60 mesh copper wire nets placed on the top and bottom surfaces, respectively, have achieved the highest $\mathrm{SE}$ of $60 \mathrm{~dB}$ in the frequency range of $9 \mathrm{kHz}$ to 1.5 GHz. It was also reported that the SE was higher when the metal nets were laminated on the surface layer than when they were laminated on the interlayer [8]. Conducting polymers, such as polyaniline and polypyrrole, were also applied in the wooden shielding material by in situ polymerization, and had an SE level above $10 \mathrm{~dB}$ in the frequency range of $100 \mathrm{MHz}$ to $3 \mathrm{GHz}$ [13]. Power coal carbon material was used to modify wood wool-cement boards. When the concentration of carbon material was $5 \mathrm{wt} \%$ and $25 \mathrm{wt} \%$, the total insertion loss of electromagnetic wave in the frequency range of $2.5 \mathrm{GHz}$ to $6 \mathrm{GHz}$ could reach 20 $\mathrm{dB}$ to $40 \mathrm{~dB}$ [14]. Carbonized wooden material, such as solid wood panel [15] and fiberboard [16] could also improve SE, which could be further improved with structural designing. It was reported that the fiberboard composite designed with the metal net and conductive coating as reflective material on the two surface layers and barite powder and magnetite powder as an absorbing material in the core layer [17] could obtain an SE of more than $60 \mathrm{~dB}$ in the frequency range of 18.85 $\mathrm{MHz}$ to $1.46 \mathrm{GHz}$. The above studies on wooden shielding material could more or less enhance SE. However, further research is required to reduce costs and weight and improve processing efficiency. 
Because of its excellent electromagnetic property and mechanical performance, as well as its low density compared with metal, carbon fiber has recently been studied as a viable material in shielding fiberboard composites [18]. The carbon fiber was mixed with wood fiber (weight ratio $3: 1$ ) to make composites reaching $47.89 \mathrm{~dB}$ in the frequency of $1 \mathrm{GHz}$. One-layer nickel-plated carbon fiber (12 k) with grid arrangement was also laminated with medium density fiberboard substrate to make composites, and were reported to achieve a SE of $41.54 \mathrm{~dB}$ to $63.73 \mathrm{~dB}$ in the frequency range of $200 \mathrm{MHz}$ to $1 \mathrm{GHz}$ [19]. Carbon fiber paper (CFP), which has a lower percentage of carbon fiber than the pure carbon fiber, low density (about $60 \mathrm{~g} / \mathrm{m}^{2}$ ), low thickness (about $0.12 \mathrm{~mm}$ ), better flexibility, good conductivity, good SE, good compressibility, good adhesion property, good permeability, and moderate price, could be a potential material for the production of laminated wooden shielding composites. Therefore, the current study was proposed: the flexible CFP was laminated with wood veneer to produce a kind of electromagnetic shielding plywood composite.

\section{TEST MATERIALS AND METHODS}

\subsection{Test Materials}

Three types of veneers, i.e., eucalyptus veneer with an average thickness of $2.4 \mathrm{~mm}$, poplar veneer with an average thickness of $1.5 \mathrm{~mm}$, and birch veneer with an average thickness of $0.4 \mathrm{~mm}$ and $0.8 \mathrm{~mm}$. All veneers were dried to a moisture content of 7 to $9 \%$ by air drying, then cut into $250 \times 250 \mathrm{~mm}$ sections.

CFP with a surface density of $60 \mathrm{~g} / \mathrm{m}^{2}$, carbon fiber concentration of about $35 \mathrm{wt} \%$ (the rest is wood fiber), and a thickness of $0.12 \mathrm{~mm}$ was supplied by the Beijing Beyond Special Materials Co. Ltd. in Beijing, China.

Powdery modified urea-formaldehyde resin adhesive (UF) with a weight ratio of powder UF (C505) to modifier (M356) to curing agent $(\mathrm{H} 271)$ to water of 100:15:15:60 was provided by Boshijiao Fendeli Adhesive Co. Ltd. of Shanghai in China.

A copper foil electrode was home-made with the following dimensions: $300 \mathrm{~mm}$ long, $10 \mathrm{~mm}$ wide, and 0.02 mm thick.

\subsection{Test Methods}

\subsubsection{Electromagnetic Shielding Composites with Different Processing Parameters}

Two groups of plywood composites were made: one for investigating the effect of pressing pressure and the other for exploring the effect of the adhesive concentration. Threelayer plywood composite was made with one-layer CFP inserted in one glue line between veneers, shown in Fig. (1). For the first group of plywood composites, the double-sided adhesive concentration was $280 \mathrm{~g} / \mathrm{m}^{2}$, the press time was 1.2 $\mathrm{min} / \mathrm{mm}$, platen temperature was $110 \pm 5{ }^{\circ} \mathrm{C}$, and pressure was at $0.8,1.0,1.2,1.6,1.8$, and $2.0 \mathrm{MPa}$, respectively. For the second group of plywood composites, double-sided adhesive concentration was 180, 230, 280, 330, 380 and 430 $\mathrm{g} / \mathrm{m}^{2}$, respectively, when the surface was bonded with the CFP, and $280 \mathrm{~g} / \mathrm{m}^{2}$ for those glue lines without CFP, press time was $1.2 \mathrm{~min} / \mathrm{mm}$, platen temperature was $110 \pm 5^{\circ} \mathrm{C}$, and pressure was at $1.2 \mathrm{MPa}$. The resistance of CFP before and after hot pressed, SE of CFP before hot pressed, the SE and the bonding strength of plywood composites were measured.

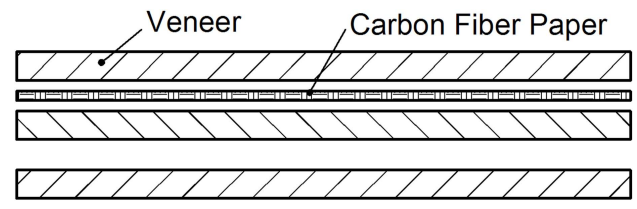

Fig. (1). Structure of three-layer plywood composite.

\subsubsection{Plywood Composites with Different Structures}

The first group of plywood composites was made as follows and the structure could be seen in Fig. (2).

Two layers of CFP were placed in different glue lines, and the double-sized spread adhesive concentration was 380 $\mathrm{g} / \mathrm{m}^{2}$ when the surface was bonded with the CFP and 280 $\mathrm{g} / \mathrm{m}^{2}$ in the glue line without CFP. The pressing time was 1.2 $\mathrm{min} / \mathrm{mm}$, platen temperature was $115 \pm 5^{\circ} \mathrm{C}$, and the pressure was $1.2 \mathrm{MPa}$. The number of veneers between two CFP layers was one (Fig. 2a), three (Fig. 2b), and five-layer veneers (Fig. 2c), respectively. SE was measured.

The second group of plywood composites was laminated with three and four layers of CFPs (Fig. 3). The other conditions were the same as in the previous group of plywood composites. SE was measured.

The third group of plywood composites was laminated with two-layer CFPs as in Fig. (2b) and Fig. (2c), but with surface-layer veneers of different thicknesses: $0.4 \mathrm{~mm}$ (birch veneer), $0.8 \mathrm{~mm}$ (birch veneer), $1.5 \mathrm{~mm}$ (polar veneer), and $2.4 \mathrm{~mm}$ (eucalyptus veneer). The other conditions were the same as in the previous group of plywood composites. SE was measured.

\subsubsection{Drop Rate of Resistance Tests}

The resistance of CFP before and after hot pressed was tested using the low-resistance testing instrument (TH2512). The drop rate of resistance was calculated as the percentage of difference between the resistance before and after hot pressed from the resistance before hot pressed.

Testing the resistance before hot pressed was done by placing two copper foil electrodes on the two ends of the CFP, as seen from Fig. (4). The two electrodes were applied with a certain pressure to impinge on the paper using a home-made clamp. The low resistance tester was used to test the resistance by connecting with the electrodes. The resistance after hot pressed was measured after the CFP with two electrodes on both ends was laminated with veneer and hot pressed.

\subsubsection{SE Performance Tests}

According to the Chinese standard "SJ20524-1995 measurement of SE of materials," the plywood composite was formed into a disc with a diameter of $115^{0}{ }_{-0.5} \mathrm{~mm}$. The SE of the plywood composite was tested using a DN15115 type of vertical flange coaxial test device [19]. The test frequency range was $30 \mathrm{MHz}$ to $1 \mathrm{GHz}$. The average value of two specimens was taken as the value of SE.

\subsubsection{Bonding Strength Tests}

The bonding strength of the plywood composite was tested in accordance with the Chinese standard "GB/T 9846- 


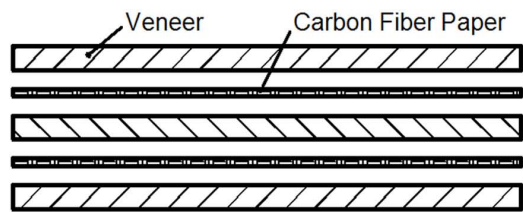

(a)

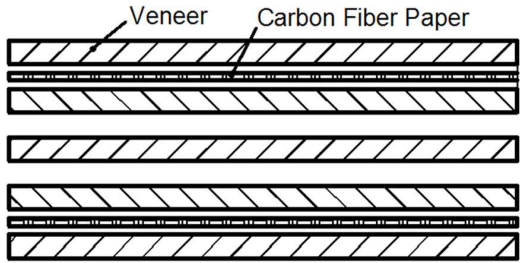

(b)

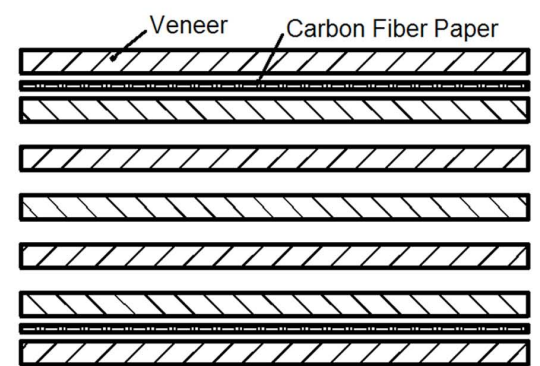

(c)

Fig. (2). Structure for plywood composites laminated with two-layer CFPs. (a) three-layer with two-layer CFPs; (b) five-layer with two-layer CFPs; (c) seven-layer with two-layer CFPs.

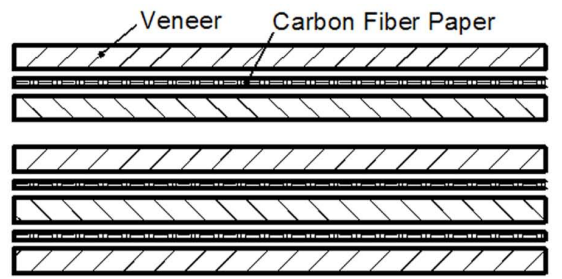

(a)

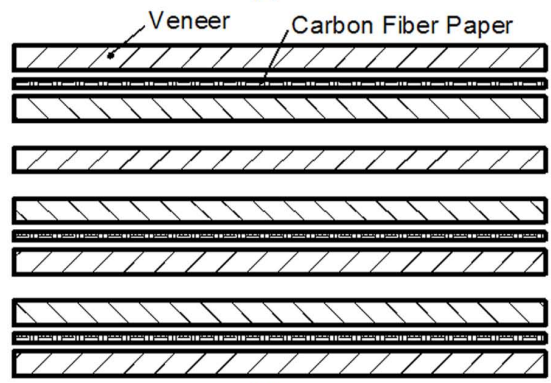

(b)

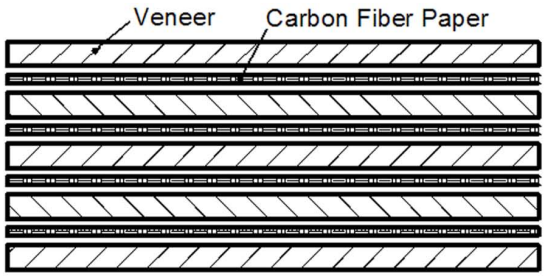

(c)

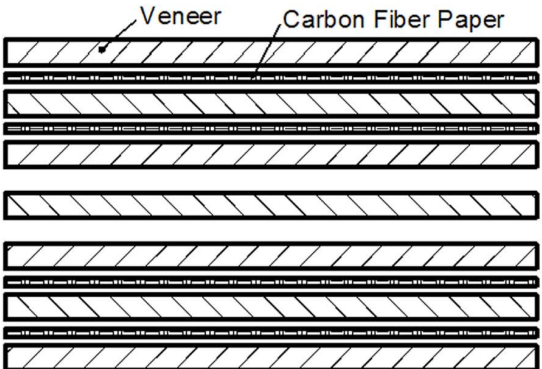

(d)

Fig. (3). Structure of plywood composites laminated with three and four-layer CFP. (a) five-layer with three-layer papers; (b) seven-layer with three-layer papers; (c) five-layer with four-layer papers; (d) seven-layer with four-layer papers.

2004 plywood" for the second type of plywood. First, the specimen was soaked in water at $(63 \pm 3){ }^{\circ} \mathrm{C}$ for three hours. Then the specimen was left to cool for ten minutes at room temperature. Finally, a universal mechanical testing machine was used to test the bonding strength of the specimen, and wood failure ratio was also recorded.

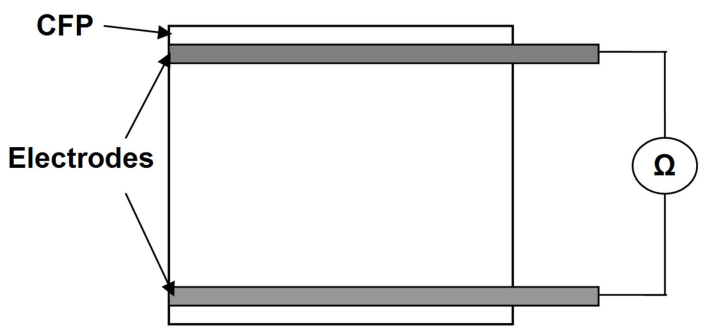

Fig. (4). Schematic diagram for testing resistance.

\section{RESULTS AND ANALYSIS}

\subsection{Microstructure of CFP}

A three-dimensional conductive net in the CFP can be clearly seen in Fig. (5), which showed that carbon fibers were staggered. As the pressure increased, those fibers, which did not contact before press, may get touch, and those in partial contact (e.g., points A, B, C in Fig. 5) and the contacted carbon fibers (e.g., point D in Fig. 5), would have increased the contact surface, resulting in a reduction of resistance. Overall, the pressure range of $1.1 \mathrm{MPa}$ to $2.1 \mathrm{MPa}$ is considered the most appropriate for the production of plywood composites, and using this parameter would give rise to a stable resistance.

\subsection{Effect of Processing Parameters on Resistance, Bonding Performance and SE}

\subsubsection{Pressure}

The reduction of resistance of CFP laminated with veneers after hot pressed is shown in Fig. (6). It can be seen that the drop rate of the resistance of the paper firstly increased, then decreased with the increase of hot press pressure as discussed in the previous section. The highest drop rate was $43.8 \%$ at a pressure of $1.8 \mathrm{MPa}$, and it was reduced to $39.8 \%$ at a pressure of $2.0 \mathrm{MPa}$. This may be because the fibers were dislocated from their original 


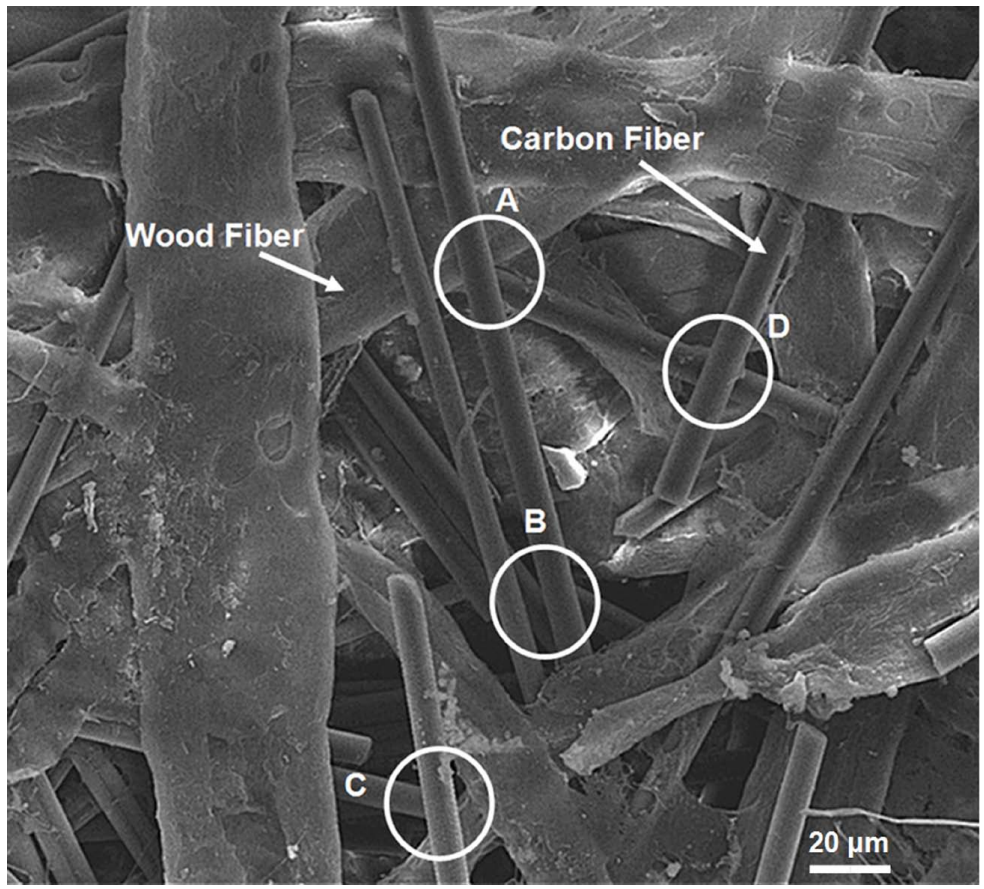

Fig. (5). SEM for CFP before hot pressed.

position to another position under sufficiently high pressure or fracture of the fibers shown as Fig. (7a), which could damage the conductive circuit composed of parts of fibers and cause the increase of resistance. However, the increased pressure may also result in closer contact among some parts of carbon fibers; therefore, the test results depend on the balance of the increase and decrease of the resistance. The above results indicate that the pressure from $1.2 \mathrm{MPa}$ to 1.8 $\mathrm{MPa}$ could be more suitable for hot-pressing plywood composites.

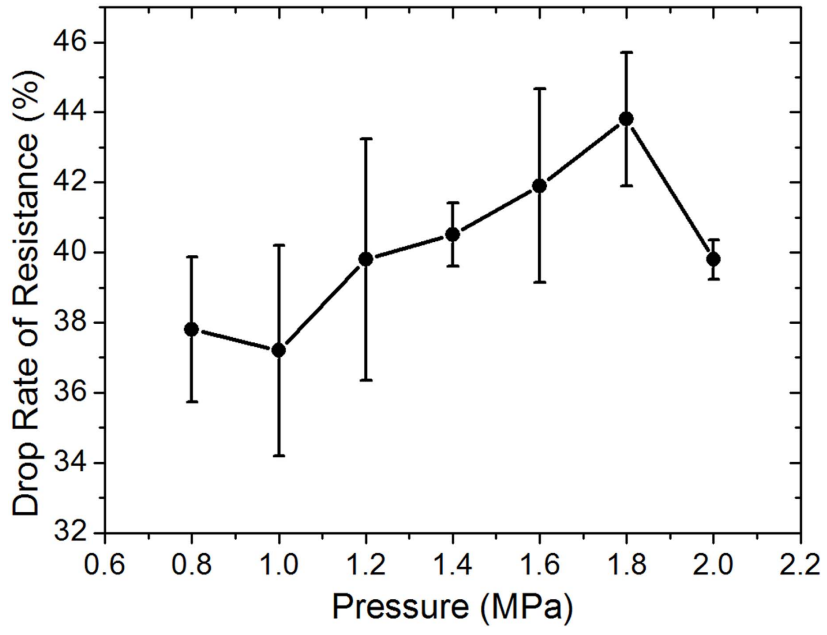

Fig. (6). Drop rate of resistance of CFP after hot pressed with different pressure.

As shown from Fig. (8), the SE of single layer CFP before hot pressed was lower than that of the plywood composite for all the frequency ranges tested in this study. More conductive micro circuitries should have been formed by carbon fiber contacted more closely after hot pressed. What is more, Fig. (7b) shows that the mesh of these circuitries become smaller and it is three-dimensional.
Generally, the mesh, contact resistance and electrical conductivity of the carbon fiber circuitries all have an impact on its electromagnetic shielding effectiveness [20]. When external electromagnetic wave are shot to the surface of three-dimensional conductive circuitries, it would inevitably cause magnetic flux change when passing through each micro circuitry, and then produce induced current and inductive magnetic field in circuits which could prevent the magnetic flux from changing in the circuit surface. According to Faraday's law of electromagnetic induction, the reversed magnetic field produced by the induced current in circuitry could offset external magnetic flux, and thereby reflect and shield external magnetic field [20]. On the other hand, the reflected wave is again reflected by other smaller circuitry in the three-dimensional conductive structure, which results in that magnetic field further weaken. Consequently, the SE after hot pressed was higher than that before hot pressed.

However, the influence of hot press pressure from 0.8 $\mathrm{MPa}$ to $2.0 \mathrm{MPa}$ on the $\mathrm{SE}$ of plywood composites was insignificant, even though there was an obvious change of resistance under different pressures shown in Fig. (6), which was further confirmed by the calculated average value of SE (Table 1). It can be seen from Table 1 that the average bonding strength of plywood composites increased with the increase of pressure from $0.8 \mathrm{MPa}$ to $2.0 \mathrm{MPa}$, more and more adhesive may penetrate into the paper and solidified by which bonding among carbon fibers can be strengthened, but stabilized afterward.

\subsubsection{Adhesive Concentration}

Fig. (9) shows that, the resistance of CFP compacted between two veneers by adhesion decreased with the increase of the adhesive concentration, with the drop rate changing from about $31.2 \%$ to $43.8 \%$ when the adhesive consumption changed from $180 \mathrm{~g} / \mathrm{m}^{2}$ to $330 \mathrm{~g} / \mathrm{m}^{2}$. 


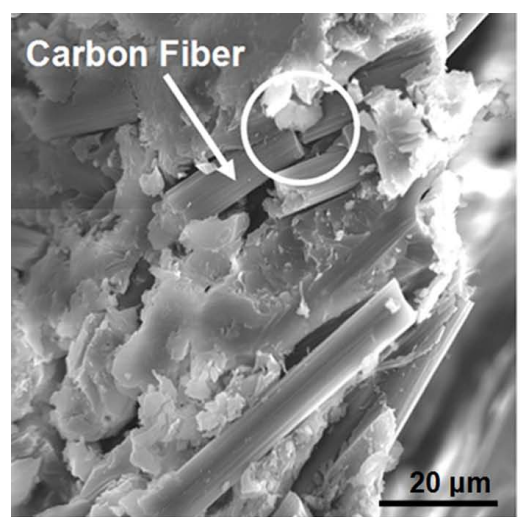

(a)

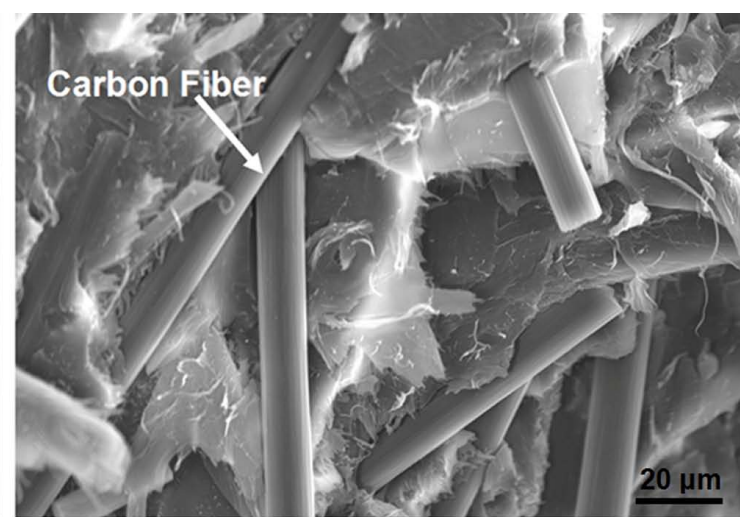

(b)

Fig. (7). Carbon fiber in the carbon fiber paper after hot pressed. (a) fracture of carbon fiber; (b) three-dimensional structure among carbon fibers in thickness.

During the hot press, the adhesive may have penetrated into the paper and solidified shown in Fig. (10b), which made the carbon fibers contact closer and not detach from each other for the compression stress after hot pressed. However, further increase of adhesive concentration resulted in a reduction of drop rate of resistance, and this may be because the excess adhesive could have penetrated into two contacted carbon fibers and resulted in the decrease of drop rate of resistance, which can be verified by the SEM in Fig. (10c) that the space among carbon fibers was apparently filled by adhesive.

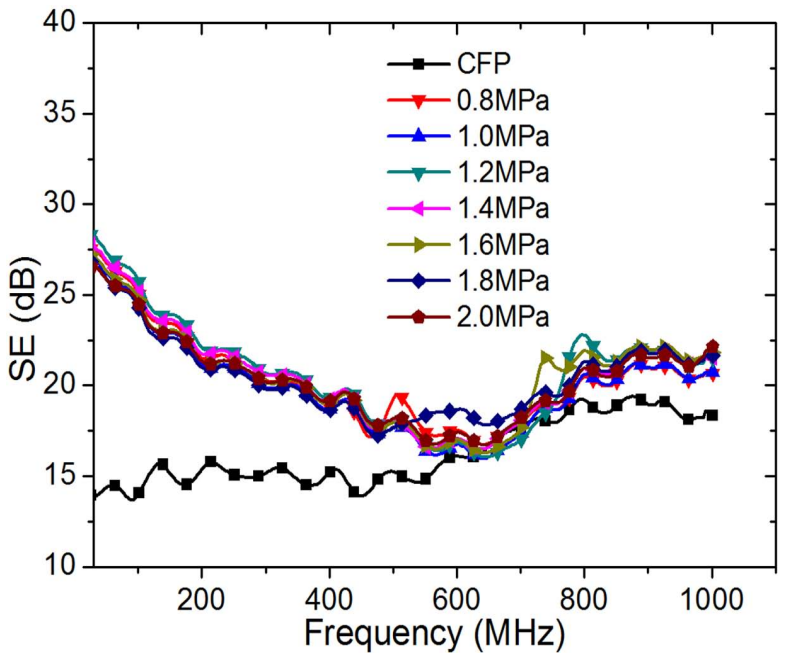

Fig. (8). SE of single layer CFP before hot pressed and plywood composite under pressure from 0.8 $\mathrm{MPa}$ to 2.0 $\mathrm{MPa}$.

Fig. (11) shows the influence of adhesive concentration from $180 \mathrm{~g} / \mathrm{m}^{2}$ to $430 \mathrm{~g} / \mathrm{m}^{2}$ on SE was not significant. The average calculated SE also supported this finding (Table 2). As for the same mechanism above, it is also found that the $\mathrm{SE}$ of single layer CFP before hot pressed was lower than that of the plywood composite for all the frequency ranges tested.

However, the bonding strength increased from $0 \mathrm{MPa}$ to $1.31 \mathrm{MPa}$ when the adhesive concentration changed from $180 \mathrm{~g} / \mathrm{m}^{2}$ to $380 \mathrm{~g} / \mathrm{m}^{2}$, and after that the bonding strength
Table 1. Average SE and bonding strength of plywood composites.

\begin{tabular}{|c|c|c|c|}
\hline $\begin{array}{c}\text { Pressure } \\
\text { (MPa) }\end{array}$ & $\begin{array}{c}\text { Average SE from } \\
\text { 30MHz to 1GHz (dB) }\end{array}$ & $\begin{array}{c}\text { Bonding } \\
\text { Strength (MPa) }\end{array}$ & $\begin{array}{c}\text { Average Wood } \\
\text { Failure Ratio (\%) }\end{array}$ \\
\hline \hline 0.8 & 19.6 & $0.87 \pm 0.42$ & 44 \\
\hline 1.0 & 19.9 & $0.84 \pm 0.32$ & 13 \\
\hline 1.2 & 20.3 & $1.07 \pm 0.25$ & 46 \\
\hline 1.4 & 20.3 & $1.11 \pm 0.30$ & 61 \\
\hline 1.6 & 20.5 & $1.34 \pm 0.24$ & 64 \\
\hline 1.8 & 20.5 & $1.01 \pm 0.23$ & 72 \\
\hline 2.0 & 20.3 & $1.29 \pm 0.06$ & 30 \\
\hline
\end{tabular}

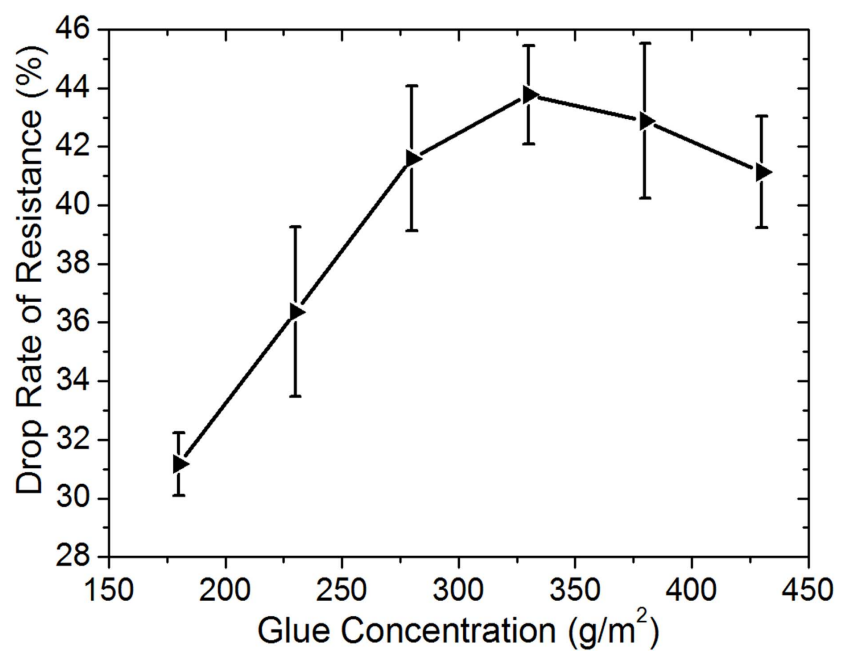

Fig. (9). Drop rate of resistance of CFP after hot pressed with different adhesive concentration.

started to decrease (Table 2). As in Fig. (10a), it is apparent that there was not enough adhesive to penetrate into the paper and the center layer of paper may not have been wellbonded by the glue when the adhesive concentration was $180 \mathrm{~g} / \mathrm{m}^{2}$. The bonding strength of the paper was very low 


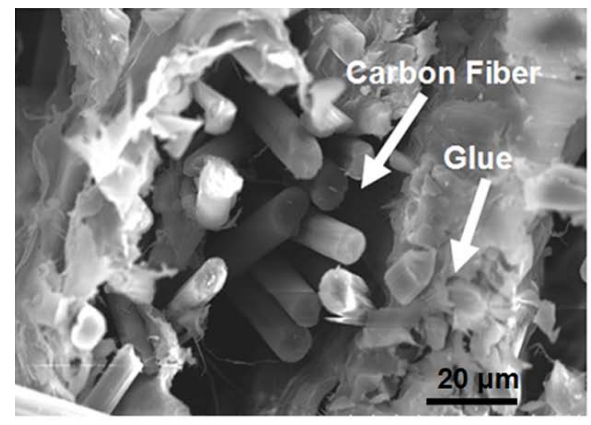

(a) $180 \mathrm{~g} / \mathrm{m}^{2}$

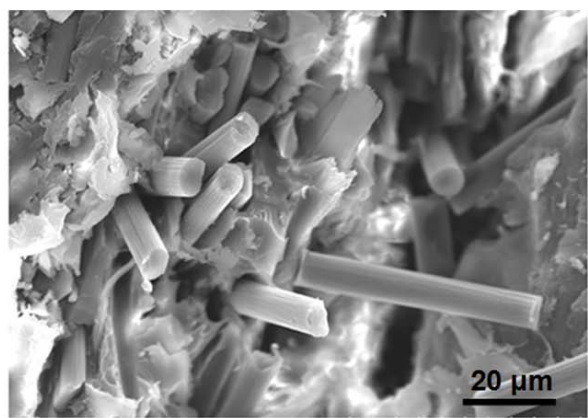

(b) $330 \mathrm{~g} / \mathrm{m}^{2}$

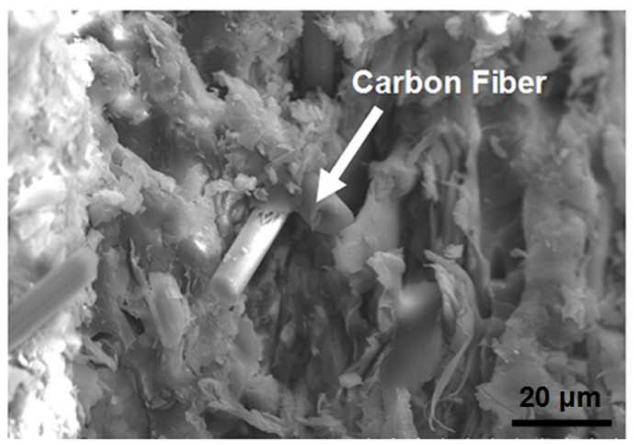

(C) $430 \mathrm{~g} / \mathrm{m}^{2}$

Fig. (10). SEM for cross section of the glued CFP with different adhesive concentration.

and hence, the specimen was easily delaminated when they were immersed in the hot water before being tested. When adhesive concentration was more than $330 \mathrm{~g} / \mathrm{m}^{2}$, adhesive fully permeated into the space among carbon fiber shown in Fig. (10b, c), consequently, the bonding strength was more credible.

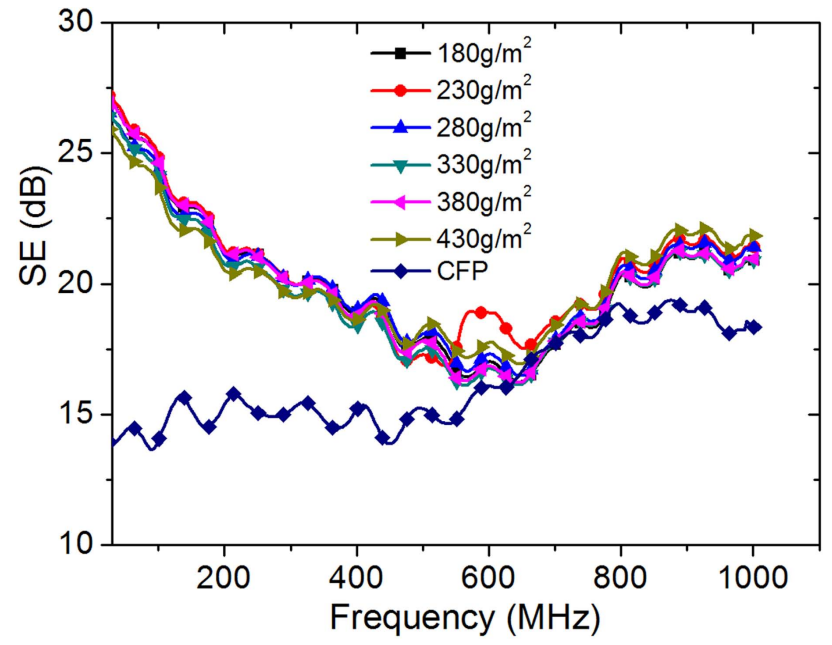

Fig. (11). SE of single layer CFP before hot pressed and plywood composite.

\subsection{Effect of CFP and Veneer Combinations on SE}

\subsubsection{Plywood Composites Laminated with Veneer of Different Thickness Between Two-layer CFPs}

Fig. (12) shows that, the number of veneer layers between two CFPs had a significant effect on SE. In the
Table 2. Average SE and bonding strength of plywood with different glue concentration.

\begin{tabular}{|c|c|c|c|}
\hline $\begin{array}{c}\text { Adhesive } \\
\begin{array}{c}\text { Concentration } \\
\left(\mathbf{g} / \mathbf{m}^{2}\right)\end{array}\end{array}$ & $\begin{array}{c}\text { Average SE } \\
\text { from } \\
\text { 30MHz to } \\
\mathbf{1 G H z}(\mathbf{d B})\end{array}$ & $\begin{array}{c}\text { Bonding } \\
\text { Strength } \\
\text { (MPa) }\end{array}$ & $\begin{array}{c}\text { Average } \\
\text { Wood } \\
\text { Failure Ratio } \\
\text { (\%) }\end{array}$ \\
\hline \hline 180 & 20.0 & 0 & 0 \\
\hline 230 & 20.4 & $0.33 \pm 0.28$ & 0 \\
\hline 280 & 20.3 & $1.07 \pm 0.25$ & 46 \\
\hline 330 & 19.7 & $1.04 \pm 0.21$ & 75 \\
\hline 380 & 20.0 & $1.31 \pm 0.28$ & 81 \\
\hline 430 & 20.1 & $1.08 \pm 0.24$ & 75 \\
\hline
\end{tabular}

frequency range of $30 \mathrm{MHz}$ to $1 \mathrm{GHz}$, the average $\mathrm{SE}$ of three kinds of plywood composite were $23 \mathrm{~dB}, 26 \mathrm{~dB}$, and $28 \mathrm{~dB}$, respectively.

Generally, reflection of electromagnetic wave occurs in interface between two kinds of shielding materials with different impedance [21]. So, the shielding change could be caused by the fact that the incident electromagnetic wave reflections were multiplied between the two-layer CFPs, which would further increase the loss of the reflected wave for certain dielectric properties of wood in the middle layer, which is shown as Fig. (13). The increase of thickness of the middle veneer would result in an increase in this loss. Similar phenomenon have also been found by Lee et al. [22], who concluded that the SE increased slowly with the increase of the thickness of L-layer (low conductive layer) in an $\mathrm{H}$ (high conductive layer)-L-H multilayer structure. The 


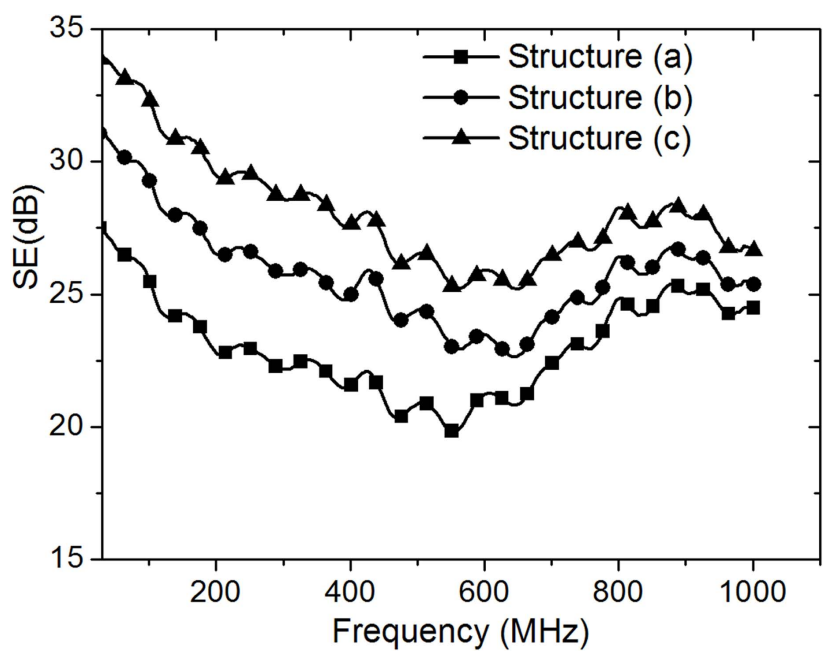

Fig. (12). SE of three different structures of plywood composite with two-layer CFPs. (a) three-layer with two-layer CFPs; (b) fivelayer with two-layer CFPs; (c) seven-layer with two-layer CFPs.

results found by Ramadin et al. [23] showed that the SE of the spaced materials with $30 \mathrm{~mm}$ between two epoxy-carbon fiber fabrics was greater than those with $0 \mathrm{~mm}, 10 \mathrm{~mm}$, and $20 \mathrm{~mm}$ spacing.

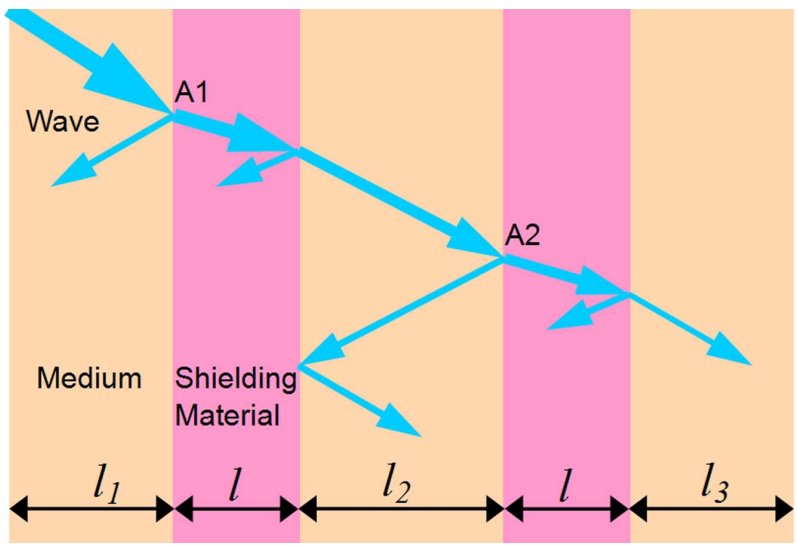

Fig. (13). Schematic diagram for double shielding (medium consists of 1, 2, 3-layer).

The shielding effect of the double-layers of composite can be further illustrated in Fig. (13), in which the doublelayers were metal shielding materials and the interlayer medium $\left(l_{1}, l_{2}\right.$, and $\left.l_{3}\right)$ is air. When shielding layers have the same thickness, the related calculation equation of total shielding effectiveness can be expressed as follows [24]:

$S E=A+R+B_{2}$

$A$ is the absorption loss, $R$ is the reflection loss, and $B_{2}$ is the multiple reflection loss.

$$
A=2 \times 8.686 l \sqrt{f \mu_{r} \sigma_{r}}
$$

$$
R=2 \times\left[20 \lg \frac{\left|1+\frac{\eta}{Z_{w}}\right|^{2}}{4 \mid \frac{\eta}{Z_{w} \mid}}\right]
$$

$$
B_{2}=201 \mathrm{~g}\left|1-q_{1} e^{-2 \gamma l}\right|+201 \mathrm{~g}\left|1-q_{2} e^{-j 2 \beta_{0} l_{2}}\right|+201 \mathrm{~g}\left|1-q_{3} e^{-2 \gamma l}\right|
$$

$f$ is the frequency, $\mu_{r}$ is the electrical constants of initial magnetic permeability, $\sigma_{r}$ is the electrical conductivity, $\eta$ is the intrinsic impedance of sheet material, $Z_{w}$ is the impedance of the incident wave, $q_{2}$ is the reflection coefficients of the shielding layer, $l$ is the thickness of the shielding layer or interlayer, $\beta_{0}$ is the phase constant of the medium, and $\gamma$ is the propagation constant of the medium.

It can be seen from Eq. (4) that $B_{2}$ is determined by the thickness $(l)$ of the interlayer. The absolute value of the middle term in Eq. (4) increases with the increase of $l_{2}$, which results in the increase of $B_{2}$ because of the increase of the distance of multiple reflections. Similarly, the increase in the SE of the plywood with the increase of thickness of interlayer between two CFPs could be ascribed to this mechanism.

The results of plywood composites laminated with threelayer CFPs, shown from Fig. (14), have further proved the influence of the thickness of space between two-layer papers on the SE, of which the SE of the (b) structure (Fig. 3) was greater than that of the (a) structure (Fig. 3) in full frequency range. The same result could also be observed with fourlayer CFPs, shown from Fig. (15).

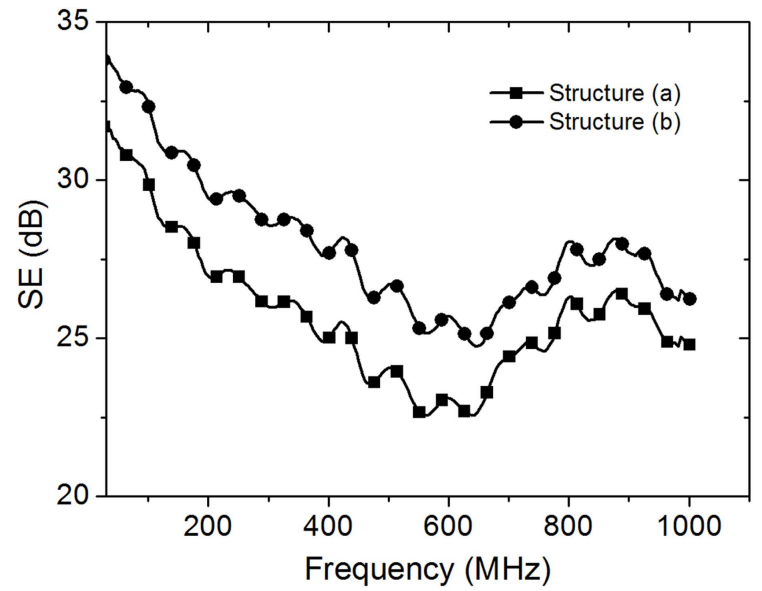

Fig. (14). SE of two different structure of plywood composites with three-layer CFPs.

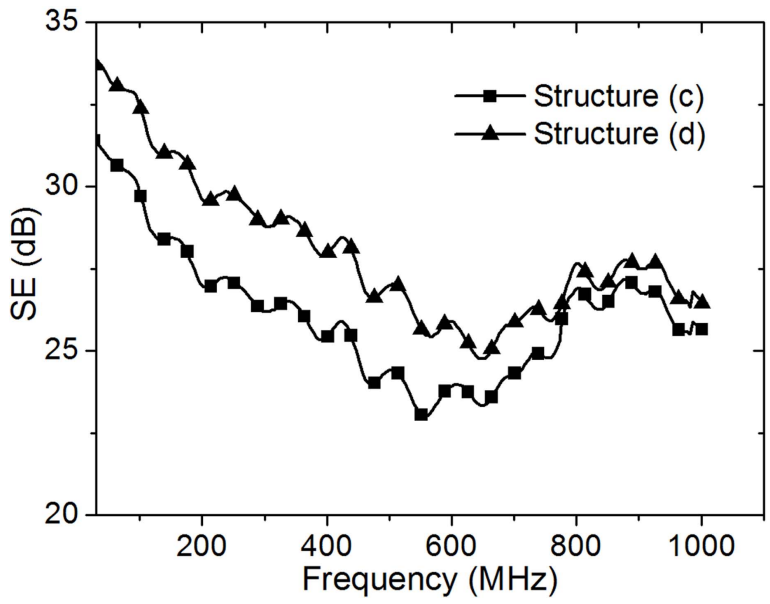

Fig. (15). SE of two different structure of plywood composites with four-layer CFPs. 


\subsubsection{Two-layer CFPs Plywood Composites Laminated with Surface Veneers of Different Thickness}

It can be seen from Figs. $(\mathbf{1 6}, \mathbf{1 7})$ that the thickness of surface veneer had a remarkable influence on its SE. The SE of plywood composites in the frequency range of $30 \mathrm{MHz}$ to $1 \mathrm{GHz}$ increased as the thickness of surface veneer decreased from $2.4 \mathrm{~mm}$ to $0.4 \mathrm{~mm}$, which could be seen in both the five-layer plywood composites and the seven-layer plywood composites. The maximum difference reached about $10 \mathrm{~dB}$. Overall, all SE in the frequency range of $30 \mathrm{MHz}$ to $1 \mathrm{GHz}$ reached above $30 \mathrm{~dB}$ when the thickness of surface veneer of five-layer plywood composites was $0.4 \mathrm{~mm}$ and that of seven-layer plywood composites was $0.4 \mathrm{~mm}$ and $0.8 \mathrm{~mm}$, which met the requirement for commercial uses [25]. The SE in the seven-layer plywood composite with a surface veneer of $0.4 \mathrm{~mm}$ thickness had a maximum of $39 \mathrm{~dB}$ and a minimum of $34 \mathrm{~dB}$ in the frequency range. The dosage of carbon fiber was only $42 \mathrm{~g} / \mathrm{m}^{2}$, much lower than in previous studies, about $430 \mathrm{~g} / \mathrm{m}^{2}$ [19].

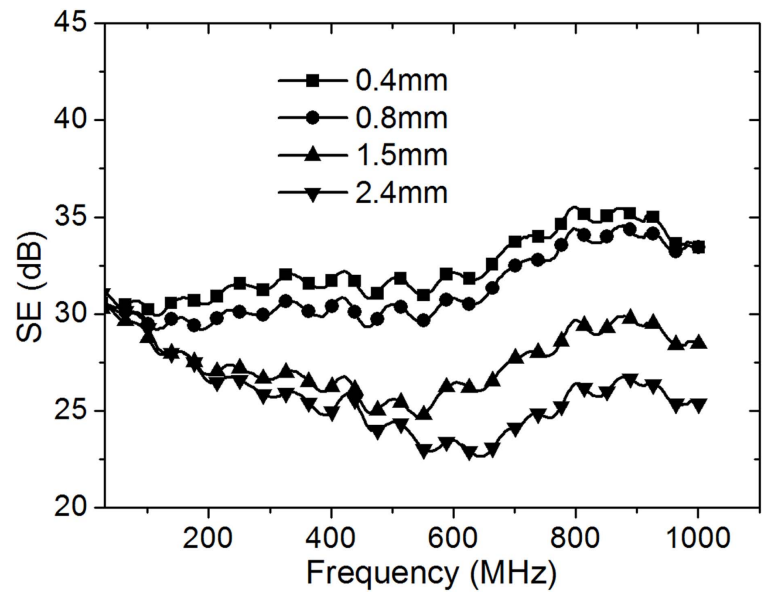

Fig. (16). SE of five-layer plywood composites laminated with surface veneer of different thickness.

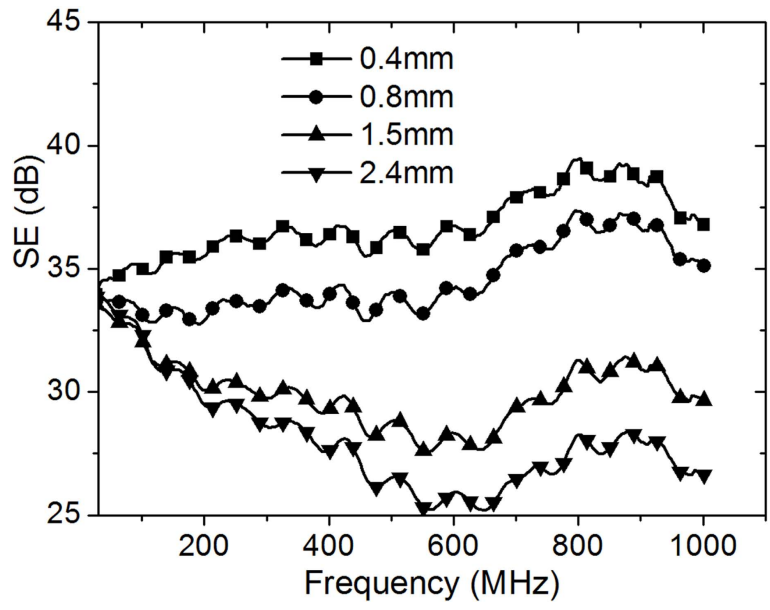

Fig. (17). SE of seven-layer plywood composites laminated with surface veneer of different thickness.

Wood material consists of polar materials, i.e., moisture, cellulose, hemicellulose, lignin, and a small amount of mineral material, and has some dielectric property [26]. Its wave impedance is greater than that of air; when an electromagnetic wave passes from air into the surface veneer, it gives rise to a refraction, the angle of which is less than that of incidence. As shown in Fig. (13), $l_{1}$ as surface veneer, also $l_{2}$ and $l_{3}$ as veneer, with the increase of the thickness of surface veneer the incident position $\left(\mathrm{A}_{1}\right)$ should move down and the incident position $\left(\mathrm{A}_{2}\right)$ also move down, so the distance of multiple reflection between two shielding material in finite width becomes shorter, which resulted in less SE.

\section{CONCLUSION}

(1) Electromagnetic shielding plywood composites laminated with CFP were investigated, illustrating that CFP is a promising material for realizing an industrial production of wooden composites with sufficient SE.

(2) It was demonstrated that CFP paper and wood veneer can appropriately laminate with a pressure of 1.2 $\mathrm{MPa}$, a modified UF adhesive concentration of 380 $\mathrm{g} / \mathrm{m}^{2}$ (surface of veneer contacted with CFP), a press time of $1.2 \mathrm{~min} / \mathrm{mm}$, and a platen temperature of $110 \pm 5{ }^{\circ} \mathrm{C}$.

(3) Both the space between two-layer CFPs in plywood composite and thickness of surface veneer had a remarkable influence on its SE, with the SE increasing as space increased and thickness decreased.

(4) The SE of the five-layer plywood composites laminated with two-layer CFPs (dosage of carbon fiber $42 \mathrm{~g} / \mathrm{m}^{2}$ ) reached above $30 \mathrm{~dB}$ in the frequency range of $30 \mathrm{MHz}$ to $1 \mathrm{GHz}$ when the thickness of its surface veneer was $0.4 \mathrm{~mm}$; and the SE of the sevenlayer plywood composites reached above $30 \mathrm{~dB}$ in the frequency range of $30 \mathrm{MHz}$ to $1 \mathrm{GHz}$ when the thickness of surface veneer was $0.4 \mathrm{~mm}$ and $0.8 \mathrm{~mm}$. Therefore, both could be applied in commercial uses.

\section{CONFLICT OF INTEREST}

The authors confirm that this article content has no conflict of interest.

\section{ACKNOWLEDGEMENTS}

The authors are grateful for the support of the Special Fund for Forest Scientific Research in the Public Welfare (China) [No: 201104004] and [No: 201404505]. We also greatly appreciate professor Mizi Fan of Brunel University in United Kingdom and professor Siqun Wang of University of Tennessee in USA for their review and suggestions.

\section{REFERENCES}

[1] Nagasawa C, Kumagai Y. Electromagnetic shielding particleboards with nickel-plated wood particle. J Wood Sci 1989; 35(12): 1092-9. Nagasawa C, Kumagai Y, Urabe K. Electromagnetic shielding effectiveness particles board containing nickel-metalized wood particles in the core layer. J Wood Sci 1990; 36(7): 531-7.

[3] Nagasawa C, Kumagai $\mathrm{Y}$, Koshizaki N, et al. Changes in electromagnetic shielding properties of particleboards made of nickel-plated wood particles formed by various pre-treatment processes. J Wood Sci 1992; 38(3): 256-63.

[4] Nagasawa C, Kumagai Y, Urabe $\mathrm{K}$, et al. Electromagnetic shielding particle board with nickel-plated wood particles. J Porous Mat 1999; 6: 247-54. 
[5] Wang L, Li J, Liu Y. Surface characteristics of electroless nickel plated electromagnetic shielding wood veneer. J Forestry Res 2005; 16(3): 233-6.

[6] Wang L, Li J, Liu Y. Preparation of electromagnetic shielding wood-metal composite by electroless nickel plating. J Forestry Res 2006; 17(1): 53-6.

[7] Liu H, Li J, Wang L. Electroless nickel plating on APTHS modified wood veneer for EMI shielding. Appl Surf Sci 2010; 257: 1325-30.

[8] Zhang X, Liu Y. Study on the wood fiber-copper wire net composite MDF. China For Prod Ind 2004; 31(5): 15-9.

[9] Zhang X, Liu Y. Study on Properties of Steel/Wood Fiber Composite MDF. China Wood Ind 2005; 19(2): 12-6.

[10] Liu X, Fu F. Stainless steel fiber/veneer composite electromagnetic-shield effectiveness Plywood. Wood Processing Machinery China 2008; 5: 22-6.

[11] Lu K, Fu F, Cai Z, et al. Study of properties of electromagnetic shielding plywood laminated with conductive sheets. J Build Mater China 2011; 14(2): 207-11.

[12] Liu X, Fu F. Study on performance of electro-conductive powder and veneer composite. J Nanjing For Univ (Nat Sci Ed) China 2009; 33(2): 95-8.

[13] Sapurina I, Kazantseva N, Ryvkina N, et al. Electromagnetic radiation shielding by composites of conducting polymers and wood. J Appl Polym Sci 2005; 95: 807-14.

[14] Makovíny I, Makovínyiová K. Shielding of electromagnetic radiation by using wood-cement boards modified with carbon in microwave frequency band. Eur J Wood Wood Prod 2011; 69: 671-

[15] Wang S, Hung C. Electromagnetic shielding efficiency of the electric field of charcoal from six wood species. J Wood Sci 2003; 49: 450-4
[16] Kwon J, Park S, Ayrilmis N, et al. Electromagnetic interference shielding effectiveness, electrical resistivity and mechanical performance of carbonized medium density fiberboard. J Compos Mater 2012; 47(16): 1951-8.

[17] Su C, Yuan Q, Gan W, et al. Study on a composite fiberboard with multiple electromagnetic shielding effectiveness. Open Mater Sci J 2012; 6: 44-9.

[18] Zhang Y, Qi W, Sun L. Charcoal composite electromagnetic shielding characteristics analysis. Forest Engineering China 2013; 29(1): 54-6, 60.

[19] Yuan Q, Su C, Huang J, et al. Process and analysis of electromagnetic shielding in composite fiberboard laminated with electroless nickel-plated carbon fiber. BioResources 2013; 8(3): 4633-46.

[20] Wan P. Primary exploration of electromagnetic shielding problems of metal nets. Safety EMC China 1999; (3): 27-9.

[21] Gan Y, Chen C. Bifurcation analysis of absorption and reflection effects in electromagnetic wave absorbing composites with multilayered structure. Appl Compos Mater 1994; 1(3): 259-63.

[22] Lee C, Song H, Jang K, et al. Electromagnetic interference shielding efficiency of polyaniline mixtures and multilayer films. Synthetic Met 1999; 102: 1346-9.

[23] Ramadin Y, Jawad S, Musameh S, et al. Electrical and electromagnetic shielding behavior of laminated epoxy-carbon fiber composite. Polym Int 1994; 34: 145-50.

[24] Schulz R, Plantz V, Brush D. Shielding theory and practice. IEEE T Electromagn C 1988; 30(3): 187-201.

[25] Du S, Wang B, Cao Y. Study of electromagnetic interference shielding effectiveness of electroconductive polymer composites. Fiber Reinforced Plastics/Composites China 2000; 6: 19-21.

[26] Sahin H, Ay N. Dielectric properties of hardwood species at microwave frequencies. J Wood Sci 2004; 35: 375-80.

(C) Yuan et al.; Licensee Bentham Open.

This is an open access article licensed under the terms of the Creative Commons Attribution Non-Commercial License (http://creativecommons.org/licenses/ by-nc/3.0/) which permits unrestricted, non-commercial use, distribution and reproduction in any medium, provided the work is properly cited. 